\title{
GOBERNANZA DEL EMPRENDIMIENTO SOCIAL: ESPECIFICACIÓN DE UN MODELO PARA EL ESTUDIO DE LA INNOVACIÓN LOCAL
}

\author{
Arturo Sánchez Sánchez 1 \\ María Luisa Quintero Soto 2 \\ Rigoberto Sánchez Rosales 3 \\ Eréndira Fierro Moreno 4 \\ Cruz García Lirios 5 \\ Universidad Autónoma del Estado de México \\ http://dx.doi.org/10.5209/NOMA.54863
}

\begin{abstract}
Resumen.- La innovación organizacional ha sido establecida a partir de la diferencia entre demandas y recursos, oportunidades y capacidades. En ese sentido, un aumento de los factores externos y una reducción de los factores internos supone un incremento de la innovación aún y cuando ésta subyace de las relaciones más que de las tareas, ya que ésta supone una inconformidad ante el clima de relaciones y el clima de tareas establecidos. Por consiguiente, el objetivo del presente trabajo fue especificar un modelo para el estudio de los procesos organizacionales en comento. Se realizó un estudio documental con una selección de fuentes indexadas a repositorios de América Latina, considerando el registro ISSN y DOI, empero el aporte del trabajo al estado del conocimiento radica en la discusión de los factores innovadores que permitan un mayor entendimiento de la conformidad y la obediencia como paradigmas limitadores del emprendimiento innovador.
\end{abstract}

Palabras claves.- Demandas, recursos, emprendimiento, innovación, conformidad

Governance of social entrepreneurship: Specification of a model for the study of local innovation

Abstract.- Organizational innovation has been established from the difference between demands and resources, opportunities and capabilities. In this sense, an increase in external factors and a reduction of internal factors supposes an increase of innovation, even when it underlies relationships rather than tasks, since this implies a disagreement with the climate of relations and the Climate of established tasks. Therefore, the objective of the present work was to specify a model for the study of the organizational processes in question. A documentary study was carried out with a selection of sources indexed to repositories in Latin America, considering ISSN and DOI, but the contribution of work to the state of knowledge lies in the discussion of innovative factors that allow a greater understanding of the conformity and Obedience as limiting paradigms of innovative entrepreneurship.

Keywords.- Demands, resources, entrepreneurship, innovation, conformity

\footnotetext{
1 Doctor en Administración, Profesor de Tiempo Completo, UAT, Tlaxcala

2 Doctora en Administración, Profesora de Tiempo Completo, UAEMEX, Nezahualcóyotl

3 Doctor en Administración, Profesor de Asignatura, UAEMEX, Coyotepec

4 Doctora en Economía, Profesora de Tiempo Completo, UAEMEX, Toluca

5 Estudios de Doctorado en Psicología, Profesor de Asignatura, UAEMEX, Huehuetoca
} 


\section{Introducción}

Roger (1983) definió una innovación como la difusión de información y sus efectos inmediatos, tempranos, tardíos y rezagados por parte de los potenciales consumidores. En ese sentido, estableció como criterio esencial de una innovación a la difusión y su inclusión en la agenda de consumidores potenciales. Es decir que una innovación no sólo es una alternativa de solución nueva, eficiente, eficaz y efectiva, sino además es un instrumento de persuasión y disuasión de otras innovaciones. Por consiguiente, los adoptadores inmediatos y tempranos de la innovación asumen más riesgos de oportunidad que los consumidores tardíos o rezagados en la adopción de la innovación.

En el marco de la difusión de innovaciones, el objetivo del presente trabajo es discutir los alcances y límites de la teoría de la difusión de innovaciones, contrastando sus postulados con la teoría de la innovación organizacional inferida a partir de los hallazgos reportados en la literatura revisada. En seguida, se especifica un modelo para el estudio de la innovación organizacional y la discusión en torno a los alcances y límites del modelo especificado.

Se llevó a cabo un estudio no experimental, transversal, exploratorio, documental y retrospectivo con una selección no probabilística de fuentes indexadas a repositorios líderes en América Latina tales como Dialnet, Latindex y Redalyc, considerando el registro ISSN y DOI como criterios de inclusión en el periodo que va de 1983 a 2016, buscando la información a partir de las combinaciones de palabras claves de innovación, conformidad, demandas, recursos y emprendimiento. La información se procesó en matrices de análisis de contenido, extrayendo las variables principales a fin de poder especificar el modelo.

\section{Teoría de la Gobernanza del Emprendimiento Social}

La innovación es"la producción o adopción, asimilación yexplotación de una novedad de valor agregado en las esferas económicas y sociales; la renovación y la ampliación de los productos,servicios y mercados; desarrollo de nuevos métodos de producción; y el establecimiento de una nueva gestiónsistemas. Es a la vez un proceso y un resultado". (Crossan y Apaydin, 2010: p. 1155).

La teoría de la innovación organizacional, siguiendo los postulados de la teoría de la difusión de innovaciones, supone una instrumentación nueva que se cristaliza en valor agregado, pero a diferencia de la difusión de innovaciones que plantea una propensión a la utilidad y los riesgos, la innovación organizacional atiende el balance entre demandas y recursos, oportunidades y capacidades (véase Figura 1). 
De este modo, la innovación organizativa es en primera instancia una difusión innovadora motivacional, instrumentada por liderazgos trasformadores, pero en la cristalización del emprendimiento, la innovación organizacional genera ventajas competitivas y valores agregados que desembocan en el reconocimiento y el prestigio.

Por consiguiente, el clima organizacional que se genere al interior de la organización propicia el surgimiento de difusiones innovadoras, aún y cuando los liderazgos nos sean trasformadores, la innovación es organizacional porque se construye a partir de las percepciones de demandas y recursos, oportunidades y capacidades.

En tal proceso, la adopción de la innovación no sólo implica su implementación, sino además su implementación ajustada a los requerimientos de talentos y liderazgos (véase Figura 2).

Es decir que la innovación organizacional, a diferencia de otras innovaciones, es una construcción permanente de las expectativas, necesidades, capacidades y oportunidades de una organización que puede asemejarse a otros conglomerados, pero adquiere un sello distintivo en la generación de creencias de rechazo o aceptación de la innovación, incluso el seguimiento de la implementación es resultado de creencias a favor o en contra de la utilidad, los costos y los riesgos de adoptar la innovación.

Por lo tanto, la teoría de la innovación organizacional no sólo anticipa escenarios de climas organizacionales en contra 0 a favor de la innovación, sino además de quienes toman las decisiones de adopción, implementación, utilización, desarrollo y evaluación.

Incluso, la teoría de la innovación organizacional predeciría la emergencia de iniciativas que cuestionen los beneficios de la innovación, así como el surgimiento de propuestas de sustitución de la innovación. En el caso de la difusión de la innovación, el clima de relaciones sería evaluado conforme al clima de tareas y no al revés.

\section{Estado del conocimiento de la innovación local}

En las organizaciones, la relación entre culturas, climas, competencias y comportamientos incide en la gestión de liderazgos, conocimientos, tecnologías y habilidades que en referencia a valores y creencias, privilegian la obtención de logros por sobre la satisfacción o la colaboración.

En el caso de las competencias éstas parecen frenar la diversidad de ideas que el alumno genera al momento de llevar a cabo una actividad (Rodríguez, 2011). En efecto, la identidad está vinculada con el acceso a información poco estructurada, pero consistente con un aprendizaje significativo. El estudiante aprende con base a un repertorio 
de símbolos y significados producidos por el contexto en el que se encuentra y traducidos por el grupo al que pertenece o quiere pertenecer (Gómez, 2010). Empero, las competencias relativas a los grupos han sido definidas a partir de una lógica individual ya que están indicadas por oportunidades, capacidades y responsabilidades inherentes a la persona más que a su interdependencia con los demás integrantes de equipo (Torelles, Coiduras, Isus, Carrera, Paris y Cela, 2011 ). Incluso, la autonomía de las instituciones incide en la elección de escuela y por consiguiente en la formación profesional a través de un curriculum particular (Prieto y Villamor, 2012). Es decir, las competencias en su modo genérico están influidas por estructuras y sistemas organizacionales que les pueden inhibir o facilitar el desarrollo de competencias más específicas. encontraron que los procesos de capacitación y adiestramiento para el desarrollo de competencias relacionadas con las ventas son inhibidas por la cultura organización tradicional.

En ámbitos tecnológicos las organizaciones desarrollan competencias relativas al empleo de ordenadores y programas que en principio los hacen más valiosos por su utilidad y más competitivos por su innovación (Secanella, 2011). En ese sentido, el desarrollo de competencias relacionadas con el liderazgo o la planificación son esenciales para entablar negocios basados en alianzas estratégicas. No obstante, el clima organizacional al verse afectado por la subcontratación de personal especializado reduce las oportunidades y con ello las capacidades y responsabilidades de los empleados cuyos puestos suponen funciones ordinarias y por ende susceptibles a su reemplazo. Es decir, a medida que las organizaciones complejizan sus procesos demandan mayores funciones a sus empleados y al acelerar la productividad y control de calidad recurren a la contratación alterna inhibiendo con ello la cultura laboral, la confianza y la lealtad de sus talentos potenciales. En este contexto, las competencias organizacionales son asumidas como habilidades más que como conocimientos y valores. Por ello, la organización pasa de ser productora de valor a reproductora de procesos reduciendo drásticamente sus ventajas competitivas. En el ámbito educativo, las competencias laborales son el resultado de una serie de procesos deliberados en los que la capacitación y el adiestramiento son instrumentos de gestión de conocimiento determinados por las demandas del mercado y el contexto económico en el que se desenvuelven las organizaciones. Empero, las oportunidades parecen derivar de escenarios en los que tanto la cultura como el clima laboral son asequibles con las capacidades y responsabilidades de sus directivos y empleados. En tal sentido, la formación de liderazgos supone la emergencia de estrategias orientadas a la consecución objetivos más que a la satisfacción laboral, aunque pueden significar procesos de emprendimiento, las organizaciones son también 
consideradas como extensiones de la cultura y en este tenor las metas están ancladas al clima de relaciones tanto como al clima de tareas.

Si la cultura y el clima organizacional a pesar de ser disímbolos, inciden en la formación profesional, entonces el aprendizaje de competencias está más orientado al saber hacer que al saber ser. En aquellas profesiones en las que las habilidades sociales e interpersonales son fundamentales para explicar la emergencia de nuevas formas de colaboración, las competencias están centradas en el aprendizaje de tecnologías y programas vinculados con la práctica profesional más que con la producción de conocimiento. Es decir, la dimensión profesional de tas competencias está enfocada en el desarrollo de técnicas de procesos que supondrían un incremento en las habilidades mientras que los conocimientos y valores permanecen sin cambios, la formación profesional de capital humano estaría definida por los avances tecnológicos más que por el desarrollo humano o la responsabilidad social y ambiental de las organizaciones. A pesar de que la optimización de procesos y minimización de residuos son consideradas ventajas competitivas, las organizaciones parecen transitar hacia la tecnificación y en consecuencia, la formación de sus talentos incluye un curriculum de habilidades más que de conocimientos o valores. En tal escenario, las organizaciones parecen generar oportunidades de capacitación y adiestramiento técnico incidiendo en las capacidades computacionales y disminuyendo la responsabilidad que implica el consumo de tecnologías y energía.

Las diferencias entre los estilos de formación profesional centrados en las habilidades más que en la producción de conocimiento o la responsabilidad social y ambiental también puede observarse en las relaciones entre directivos y empleados. En el marco de las instituciones educativas las diferencias organizacionales se traducen en diferencias de aprendizaje en cuanto a competencias se refiere. Así de este modo los líderes tienden a resaltar más los logros que el aprendizaje de habilidades o el respeto a los valores de la organización. En contraste, los empleados enfatizan más el aprendizaje de estrategias en cuanto a la eficiencia de procesos más que al logro de metas o la implementación de una cultura laboral. Por su parte los directivos que enaltecen los valores de la empresa tienden a minimizar los programas de adiestramiento y capacitación, aunque los empleados adquieran una serie de técnicas y estrategias, o en todo caso cumplan con los propósitos de colaboración, productividad, calidad y competitividad, la gestión de una identidad prolifera sobre la producción de conocimiento (Genesí, Romero y Tinedo, 2011). En tal sentido, las organizaciones se diferencian por la formación de su capital humano y su enfoque en tres dimensiones de competencias:

- $\quad$ Lo que la organización desea o quiere hacer alude a principios normativos en los que la cultura organizacional predispone a los 
directivos y empleados a considerar las tradicionales formas de producción que son vistas como modelos a seguir siempre y cuando se asemejen a los estilos de liderazgo y comportamiento organizacional dominantes en las empresas.

- $\quad$ Lo que la organización sabe hacer y que supone un conjunto de habilidades y conocimientos sustentados en valores y creencias que al incidir en decisiones de producción, calidad o competitividad restan importancia a la generación de conocimiento o la gestión de tecnologías y procesos.

- Lo que la organización es capaz de hacer y por ende está delimitada por la producción de conocimiento más que por su gestión, la formación de su capital humano más que la satisfacción de sus talentos y establecimiento de metas antes de cumplir con objetivos así como la posibilidad de cambios antes de que los propósitos y las estrategias se agoten.

Las organizaciones que desean, saben y son capaces de llevar a cabo estilos de liderazgo y elaboración de procesos están más cercanas a una cultura organizacional en la que las competencias son recursos de cambio más que de conformidad o satisfacción (Henao y Londoño, 2012: 204).

En síntesis, las competencias organizacionales parecen vincular a las normas, valores y creencias con los conocimientos y habilidades que indicarían una cultura organizacional centrada en los procesos más que en la producción y gestión de conocimiento, empero las competencias resaltan el clima de tareas como su sello distintivo ya que la implementación de tecnologías implica procesos y programas de adiestramiento y capacitación, las habilidades más que los valores describirían organizaciones tecnificadas acordes a la demanda del mercado en detrimento de su planificación, estrategias y alianzas las cuales sólo sería intermediarías del propósito esencial de insertar a la organización al concierto global. No obstante, en el ámbito local, la responsabilidad social y ambiental está inmersa en los requerimientos que las trasnacionales solicitan a sus maquiladoras y distribuidoras mientras que la tecnificación de procesos sólo es un valor agregado a los sistemas de gestión, la cultura empresarial de las pymes devela su querer hacer más que su saber hacer o su capacidád de hacer.

\section{Especificación de un modelo para el estudio de la innovación local}

El modelo incluye cuatro hipótesis explicativas de los procesos organizacionales en torno a la gobernanza de las innovaciones. Se trata de un proceso en el que el seguimiento y evaluación constante por parte de talentos y líderes en torno a la innovación que en sí ya supone un proceso de emprendimiento no equiparable a la adopción, sino más 
bien al establecimiento de una agenda. Esto es así porque la gobernanza supone un diálogo constante entre los actores, así como una corresponsabilidad entre las partes.

Hipótesis 1: De la innovación a su difusión. El emprendimiento es una instancia que está asociada a la agenda organizacional. En ese sentido, la innovación es un tema central en tanto genera opiniones a favor y en contra de su implementación porque anuncia el cambio organizacional y con ello el surgimiento de la resistencia al cambio. Por consiguiente, el emprendimiento de una innovación es un reto que los actores asumen como amenaza o como oportunidad en el marco del cambio organizacional.

Hipótesis 2: Del emprendimiento a la adopción a través de la difusión. Si el balance organizacional permite la aceptación e implementación de una tecnología, entonces los grupos a favor de la utilidad toman decisiones con un alto riesgo las cuales estarían influidas por la motivación de líderes transformadores más que autoritarios, empero el emprendimiento de una tecnología o proceso puede implementarse a través de la imposición más que de la negociación o la consulta.

Hipótesis 3. De la innovación a la implementación. Si el clima organizacional de tareas prevalece sobre el clima organizacional de relaciones, entonces se trata de un caso de emprendimiento e implementación forzados en los que talentos y líderes carecen de voz y voto, siguiendo la directrices pre-establecidas, el proceso está determinado sin considerar la difusión para llegar a la adopción. Se trata de un modelo en el que los riesgos son asumidos por quien toma la decisión de implementar la innovación tecnológica o la innovación procesual.

Hipótesis 4. De la innovación a la evaluación a través de la difusión y la adopción, o bien, a través de la implementación. En la primera opción, la decisión de consensuar la adopción de una tecnología o proceso supone una corresponsabilidad en el establecimiento de una agenda para evaluar los costos y beneficios, alcances y límites del acuerdo entre talentos y líderes.

Sin embargo, cuando más bien la decisión de implementar la tecnología o proceso es asumida unilateralmente, entonces la autoevaluación prevalece sobre cualquier propuesta de mejora al sistema tecnológico o procesual adoptado.

De este modo, el estudio de la innovación organizacional puede llevarse a cabo a partir de la contrastación de las hipótesis relativas a las trayectorias de relaciones de dependencia entre el emprendimiento, la difusión, la adopción, la implementación y la evaluación de un sistema tecnológico o proceso organizacional que repercuta en la generación de ventajas competitivas y valores agregados. 


\section{Consideraciones finales}

Las culturas organizacionales en referencia a las competencias laborales están vinculadas por climas de colaboración y tareas que reflejan la influencia de los contextos en los que tanto pymes como trasnacionales se ven expuestas en su afán de internalización para las primeras e incursión en los mercados locales para el caso de las segundas.

Si se considera que la Globalización es un contexto en el que lo global y lo local se vinculan a través de las organizaciones trasnacionales en alianza con las pymes, entonces se abre la posibilidad de discutir las implicaciones que tal contexto tiene en la formación del capital humano y sus competencias laborales.

El presente escrito tiene el propósito de exponer las relaciones entre cuadros directivos en empleados en el marco de las culturas organizaciones con énfasis en la formación de sus competencias laborales y sus implicaciones para la responsabilidad social y ambiental. Cabe anticipar que no se trata de una revisión exhaustiva acerca de los conceptos relativos a cultura y competencias, ni siquiera al análisis de las competencias como factores de producción o competitividad, aunque sus definiciones supongan que han sido implementadas como instrumentos para el logro de objetivos, más bien el presente trabajo sólo expone algunos elementos en los que las culturas organizacionales parecen vincularse con las competencias específicas de cuadros directivos y empleados. En ese aspecto, se advierte una diferencia sustancial entre lo que las organizaciones desean, saben y son capaces de hacer.

Se parte del cuestionamiento y el supuesto según los cuales las organizaciones, en tanto entramados de conocimientos, habilidades y valores, permiten la construcción de una responsabilidad social y ambiental, pero al mismo tiempo están determinadas por climas de relaciones y tareas que subsumen sus tradiciones. Ello impediría a las empresas socialmente responsables transitar hacia una cultura sustentable indicada por competencias en perfecto balance, sin embargo, lejos están las pymes de insertarse en el concierto global y las trasnacionales de participar en el mercado local ya que sus productos carecen de valor en el sentido cultural más que económico. Es decir, a medida que las organizaciones han privilegiado la formación de competencias centradas en el adiestramiento y capacitación de procesos, están expuestas a elaborar productos y ofrecer servicios que desvinculen a sus clientes de ellas mismas. Tal fenómeno no sólo implica deslealtad o desconfianza, sino la pérdida de responsabilidad ante el contexto que las provee de recursos y capitales.

En el futuro, la formación del capital humano deberá circunscribirse a la adquisición de valores, normas y creencias que vinculen a las 
organizaciones con los consumidores. De lo contrario, éstas las empresas tecnificadas y reproductoras de modelos, conocimientos y procesos aspirarán a vender sólo a un sector pudiente que les exigirá aún más innovaciones y valores agregados sin garantizarles su lealtad.

La Globalización económica supone una serie de lineamientos organizacionales que impactan a los sistemas educativos principalmente a aquellos en los que la implementación de competencias indica un desarrollo laboral. Antes bien, las organizaciones al contener al capital humano construyen un arquetipo de sí mismas conocido como cultura laboral.

A partir de la cultura organizacional es posible identificar cuatro dimensiones relacionadas con la formación profesional y el desarrollo de competencias organizacionales: cooperacracia, adhocracia, competicracia, pretocracia y mercadocracia. La primera alude a la colaboración indicada por su grado de compromiso, la segunda sugiere una dinámica hedonista en la que sus líderes son creativos, la tercera se circunscribe a las competencias en el sentido de producción de conocimiento para la eficiencia de procesos que por su grado de especialización requieren de líderes formados en un conocimiento técnico. La cuarta dimensión retoma al orden como su sello distintivo y a su liderazgo conservador, por último, la quinta dimensión está ajustada a la dinámica del mercado ya que sus líderes buscan a toda costa cumplir con los objetivos establecidos.

La cultura laboral enmarca una serie de principios que guían las relaciones entre los individuos en referencia a sistemas económicos, políticos o sociales. A medida que las organizaciones se circunscriben a procesos de globalidad o localidad emergen estilos de liderazgo que ajustan los objetivos de las organizaciones a los parámetros de competitividad que el contexto les demanda. No obstante que los individuos y los grupos a los que pertenecen han adoptado y perfeccionado actitudes, decisiones y acciones, los propósitos organizacionales al estar vinculados con las estructuras sociales, los sistemas económicos o los regímenes de gobierno influyen en la construcción de empresas y sus formas de organización.

Las implicaciones que la producción y gestión del conocimiento organizacional tiene para la responsabilidad social y sustentable son diversas si se considera que la cultura y el clima laboral son ejes transversales en los que las competencias se gestan y ajustan a las pymes al mercado global mientras que insertan a las trasnacionales al ámbito local. A medida que las organizaciones transitan de un querer hacer a una capacidad de hacer limitan la planificación y flexibilizan sus procesos a fin de que la formación profesional sus talentos se aproximen al emprendimiento más que a la reproducción de conocimiento. En este escenario, las culturas organizacionales transforman sus conocimientos en habilidades ya que la entrada de 
tecnología implica una reproducción de procesos que la capacitación y el adiestramiento pueden solventar. Sin embargo, las competencias laborales al tener su origen en climas organizacionales poco estructurados propician que las diferencias salariales entre directivos y empleados se acentúen ahora en términos culturales. En efecto, las organizaciones orientadas al logro parecen soslayar la iniciativa y el compromiso individual o grupal para enaltecer las metas como las principales áreas de oportunidad que implicarían paradójicamente,capacidades relativas a producción de conocimiento, innovación tecnológica o procesos competitivos.

Es así como las culturas organizacionales se aproximan a la responsabilidad social y ambiental no como el resultado de una gestión de conocimiento o el procesamiento de saberes, sino a partir de vicisitudes derivadas de las diferencias entre ámbitos globales y locales, trasnacionales y pymes, cuadros directivos y empleados o a nivel personal, las diferencias entre valores, habilidades y conocimientos.

Precisamente, las organizaciones han sabido navegar en la tempestad que implican los mercados y los retos de sus competidores, incluso han establecido alianzas que las obligan a dividir, muchas veces compartir sus ganancias más que sus pérdidas, han ajustado sus recursos y capitales para satisfacer a sus clientes y socios, pero han entendido que la responsabilidad social y ambiental sólo se limita a la producción más que a la gestión y es en la construcción de sus culturas donde sus valores han perdido relevancia frente a las habilidades. En tal esquema, las competencias se han transmutado en prácticas y procesos que suponen la reproducción de conocimiento más que la innovación y la autonomía, las organizaciones se dirigen hacia la conformidad y la dependencia. La reproducción de modelos organizacionales, planificaciones estrategias y adiestramiento de procesos indica el estado en el que las organizaciones se encuentran y el futuro que les espera mientras no modifiquen su cultura; valores, normas y creencias.

En un escenario en el que las competencias son el resultado de la promoción de valores, la producción de conocimiento y la formación de habilidades, las culturas organizacionales se encontrarían en perfecto equilibrio con el clima laboral, la inversión en tecnología y la formación de capital humano. En contraste, en el escenario hacia el que se aproximan las organizaciones que reducen sus culturas a simples protocolos de convivencia las competencias son sólo habilidades que conllevan insatisfacción y deslealtad.

La especificación de la cultura organizacional como un factor de competencia de propensión al futuro,la orientación a la sustentabilidad es el factor determinante de Desarrollo Local. En este sentido, los deseos, saberes y capacidades fungen como condicionantes del emprendimiento de proyectos de fomento empresarial. 
En el marco del desarrollo comunitario, la cultura organizacional es un factor que inhibe o petencializa la inserción de las empresas locales en el mercado global, así como la inclusión de las trasnacionales en el mercado local.

En el marco del Desarrollo Local en donde prevalecen alianzas estratégicas entre micros, pequeñas y medianas empresas con respecto a trasnacionales, la imposición de tecnologías y procesos es una práctica común, pero cada vez más una creciente adopción de innovaciones supone una negociación y corresponsabilidad interna en las mipymes que se refleja en sus estrategias de negocios con trasnacionales, En este sentido, el presente trabajo ha especificado un modelo a partir de los marcos teóricos, conceptuales y empíricos de la literatura revisada a fin de poder estudiar la innovación organizacional desde cuatro hipótesis que al ser contrastadas indicarían el grado de innovación organizacional y las áreas de oportunidad subsecuentes.

Sin embargo, la especificidad de cada organización obliga a la inclusión de otros factores tales como competitividad o calidad que la literatura reporta como variables espurias que pueden no tener efecto alguno sobre las variables incluidas en el modelo, pero que en la situación particular de las mipymes determinaría una adopción, implementación o evaluación.

\section{Referencias}

Abdiaziz, M. y Yassin, A. (2014). "Corporate innovation and organizational performance: the case of Somalia telecommunication industry". Proceding Kuala Lumpur International Business, Economics and Law Conference, 4 (1), 260-271

Crossan, M. y Apaydin, M. (2010). "A multi-dimensional framework of organizational innovation: A systematic review of literature. Journal of Management Studies, 47 (6), 1154-1191 [DOI: 10.1111/j.1467-
[ 6486.2009.00880.x]

Genesí, M.r. Romero, N. y Tinedo, Y. (2011). "Comportamiento organizacional del talento humano en las instituciones educativas". Negotium, 18, 102-128

Gómez, F. (2010). "Competencias profesionales en Trabajo Social". Portularia, 10, 51-63

González, R. y García, F. (2011). "Innovación abierta: un modelo preliminar desde la gestión del conocimiento". Inteligible Capital, 7 (1), 82-115 [DOI: 10.3926/ic.2011.v7n1.p82-115]

Henao, R. y Londoño, A. (2012). "Diseño de un modelo de dirección por competencias distintivas para las pymes exportadoras". SemestreEconómico, 15, 197-223 
Jalonen, H. (2012). "The uncertainty of innovation: a systematic review of the literature. Journal of Management Research, 4 (1), 1-47

Lewandosky, M. (2013). "How to monitor the effects of managerial innovation in public cultural institutions. Knowledge Management \& Innovation, 19-21 june 2013, Zaclar Croatia

Luoma, V., Lappalainen, R., Uusitado, O., Vos, M., Lamsa, A. y Maaranen, P. (2012). "Added value of intangibles for organizational innovation." Human technology, 8 (1), 7-23 [DOl: org/10.17011/ht/urn.201205141650]

Marques, J. (2014). "Closed versus open innovation: evolution or combination?" International Journal of Business and Management, 9 (3), 196-203[DOl: i.org/10.5539/ijbm.v9n3p196]

Prieto, M. y Villamor, P. (2012). "Libertad de elección, competencia y calidad". Revista de Curriculum y Formación del Profesorado, 16, 150162

Roger, E. (1983). Theory of innovation difusión. New York: Prentice Hall Rodríguez, J. (2011). "Los rincones de trabajo en el desarrollo de competencias básicas". Revista Docencia e Investigación, 21, 105-130

Rodríguez, R., Moreno, B., Rivas, S., Álvarez, A. y Sanz, A. (2010). "Positive psychology at work: mutual gains for individuals and organizations. Revista de Psicología del Trabajo y las Organizaciones, 26 (3), 235-253 [DOI: 10.5093/tr2010v26n3a7]

Rodríguez, R. y Hechanova, M. (2013). "A study of cultura dimensions, organizational ambidexterity, and perceived innovations in team." Nournal of Technology Management and Innovation. 9 (3), 21-33

Secanella, J. (2011). "Nuevas competencias para la empleabilidad de los estudiantes universitarios". Cuestión Universitaria, 7, 40-47

Torelles, C., Coiduras, J., Isus, S., Carrera, J., Paris, G. y Cela, J. (2011). "Competencia de trabajo en equipo: definición y categorización". Revista de Curriculum y Formación del Profesorado, 15, 329-344

Urbancova, H. (2013). "Competitive advantage achievement through innovationand knowledge". Journal of Competitiveness, 5 (1), 82-96 [DOI: $10.7441 /$ joc.2013.01.06] 
ANEXO

Tabla 1. Conceptos básicos

\begin{tabular}{|c|c|c|c|c|}
\hline Constructo & Definición & Autor & Año & Página \\
\hline Aim of innovation. & $\begin{array}{l}\text { "is the overall result that the } \\
\text { organization want to achieve through } \\
\text { innovation." }\end{array}$ & Luoma et al. & 2012 & 11 \\
\hline Ambidexterity. & $\begin{array}{l}\text { "is defined as the ability of leaders, } \\
\text { teams, and individuals to be both } \\
\text { explorative and exploitative. } \\
\text { Explorative behavior is characterized } \\
\text { by creativity, adventure, and risk } \\
\text { taken. A person in a explorative } \\
\text { mode produces a myriad divergent } \\
\text { ideas and as many as possible } \\
\text { alternatives to problem salving or } \\
\text { opportunity seeking (...) results } \\
\text { orientation, systematization and } \\
\text { efficiency." }\end{array}$ & $\begin{array}{l}\text { Rodríguez y } \\
\text { Hechanova }\end{array}$ & 2013 & 22 \\
\hline Clarifying & $\begin{array}{l}\text { "Means the detailed definition of the } \\
\text { relationship between the organization } \\
\text { and innovation". }\end{array}$ & Jalonen & 2012 & 4 \\
\hline $\begin{array}{l}\text { Consequences } \\
\text { uncertainty. }\end{array}$ & $\begin{array}{l}\text { "innovations may have indirect, } \\
\text { undesirable and unanticipated } \\
\text { consequences. Even thought the } \\
\text { majority of the literature is focused on } \\
\text { detrimental indirect and } \\
\text { unanticipated consequences. It is } \\
\text { important to note that indirect and } \\
\text { anticipated yet also positive } \\
\text { consequences my increase } \\
\text { uncertainty." }\end{array}$ & Jalonen & 2012 & 33 \\
\hline Creative destruction. & $\begin{array}{l}\text { "Understood a seeing and doing } \\
\text { things differently implies that } \\
\text { innovation is a specific form of } \\
\text { change process." }\end{array}$ & Jalonen & 2012 & 4 \\
\hline Implementation. & $\begin{array}{l}\text { "Refer to deciding between } \\
\text { alternatives and putting innovation to } \\
\text { use". }\end{array}$ & Jalonen & 2012 & 3 \\
\hline Initiation. & $\begin{array}{l}\text { "Refers to identifying problems, } \\
\text { evaluating alternatives." }\end{array}$ & Jalonen & 2012 & 3 \\
\hline Innovation. & $\begin{array}{l}\text { "is defined as both the generation } \\
\text { and implementation of new ideas, } \\
\text { one construct that has come to fore } \\
\text { to describe the ability to do both is } \\
\text { organizational ambidexterity." }\end{array}$ & $\begin{array}{l}\text { Rodríguez y } \\
\text { Hechanova }\end{array}$ & 2013 & 22 \\
\hline Innovation. & $\begin{array}{l}\text { "An introduction of a new production } \\
\text { method, product or its quality, the } \\
\text { opening up of a new market or a } \\
\text { new source for raw materials or semi- } \\
\text { manufactures, or the creation of a }\end{array}$ & Lewandosky & 2013 & 560 \\
\hline
\end{tabular}




\begin{tabular}{|c|c|c|c|c|}
\hline & $\begin{array}{l}\text { new organizational structure in } \\
\text { industry." }\end{array}$ & & & \\
\hline Innovation. & $\begin{array}{l}\text { "As a process to inherently involve } \\
\text { unknown uncertainty". }\end{array}$ & Jalonen & 2012 & 7 \\
\hline Innovation. & $\begin{array}{l}\text { "Meanwhile, is taken as the first } \\
\text { commercialization of a new product, } \\
\text { process or system corresponding to } \\
\text { the introduction on the market of the } \\
\text { conversion (into a good service) of } \\
\text { such innovation." }\end{array}$ & Marques & 2014 & 197 \\
\hline $\begin{array}{l}\text { Innovations } \\
\text { characteristics. }\end{array}$ & $\begin{array}{l}\text { a strong relationships between } \\
\text { market performance a new products. } \\
\text { New products help maintain market } \\
\text { shares and improve profitability. } \\
\text { Growth also be means of non price } \\
\text { factors. Ability to substitute outdate } \\
\text { products, of processes that lead to } \\
\text { production time shortening and } \\
\text { speed up new product development } \\
\text { in comparison to competitors." }\end{array}$ & Urbancova & 2013 & 84 \\
\hline Innovative capability. & $\begin{array}{l}\text { "highlights the likelihood that } \\
\text { organization produces innovative } \\
\text { outcomes.. the more diverse the } \\
\text { human resource are, the larger the } \\
\text { pool of skills and perspective } \\
\text { available to the organization, the } \\
\text { more creative and innovative this } \\
\text { pool of individuals, and the higher the } \\
\text { likelihood or generating peak levels of } \\
\text { performance." }\end{array}$ & Luoma et al. & 2012 & 14 \\
\hline Innovative culture. & $\begin{array}{l}\text { "whiting and organization has a } \\
\text { strongly positive effect on } \\
\text { organization innovation because it } \\
\text { motivates people worked whiting the } \\
\text { organization to ideal with novelty, } \\
\text { individual initiatives, and collective } \\
\text { actions, while equally shaping their } \\
\text { understanding and behavior in } \\
\text { regard to risk and opportunities." }\end{array}$ & Luoma et al. & 2012 & 8 \\
\hline Intangible assets. & $\begin{array}{l}\text { "refer to something indefinite and } \\
\text { incapable or being perceived by the } \\
\text { senses:-intangible lack physically } \\
\text { substance or intrinsic productive } \\
\text { value, yet there are saleable, } \\
\text { although not materially or physically. } \\
\text { (...) are long-lived and have } \\
\text { measurable value; trust, ideas, skills, } \\
\text { reputation, process, established social } \\
\text { networks, patents, trademarks and } \\
\text { marks." (p. 9). }\end{array}$ & Luoma et al. & 2012 & 9 \\
\hline Invention. & $\begin{array}{l}\text { "Regardless of the scientific or } \\
\text { empirical origin if consist of an idea or }\end{array}$ & Marques & 2014 & 196 \\
\hline
\end{tabular}




\begin{tabular}{|c|c|c|c|c|}
\hline & $\begin{array}{l}\text { scheme, of foreseeing the possibility } \\
\text { of a new product, process or system." }\end{array}$ & & & \\
\hline Legitimacy uncertainty. & $\begin{array}{l}\text { "when necessary skills skill and } \\
\text { knowledge contradict the existing skill } \\
\text { and knowledge possessed by user } \\
\text { (...) when that innovation threatens } \\
\text { and individual's basic values and(or } \\
\text { organization norms". }\end{array}$ & Jalonen & 2012 & 28 \\
\hline Liderazgo. & $\begin{array}{l}\text { "es un proceso a través del cual el } \\
\text { líder ejerce una influencia en un } \\
\text { grupo de individuos con la intención } \\
\text { de que sus seguidores trabajen en } \\
\text { pro de un objetivo común." }\end{array}$ & $\begin{array}{l}\text { González y } \\
\text { García }\end{array}$ & 2011 & 91 \\
\hline Managerial uncerfainty. & $\begin{array}{l}\text { "in innovation manifest itself as a fear } \\
\text { of failure and as a lack of the tools } \\
\text { required to manage the risk inherent } \\
\text { in innovation processes." }\end{array}$ & & 2012 & 30 \\
\hline Market uncertainty & $\begin{array}{l}\text { "in innovation exist on the hand, due } \\
\text { to unforeseeable changes in relation } \\
\text { between firms and customer and, on } \\
\text { the other hand, due to unforeseeable } \\
\text { changes in relation between } \\
\text { competitors from which new markets } \\
\text { emerge". }\end{array}$ & Jalonen & 2012 & 24 \\
\hline Matching. & $\begin{array}{l}\text { "refers to aligning a problem } \\
\text { associated with the organization`s } \\
\text { agenda with an innovation." }\end{array}$ & Jalonen & 2012 & 4 \\
\hline Means of innovation. & $\begin{array}{l}\text { "refers to the necessary resources } \\
\text { that need to be in place for } \\
\text { innovation." }\end{array}$ & Luoma et al. & 2012 & 11 \\
\hline Innovación abierta. & $\begin{array}{l}\text { "el uso de flujos internos y externos de } \\
\text { conocimiento de forma determinada } \\
\text { para acelerar la innovación interna y } \\
\text { expandir los mercados para el uso } \\
\text { externo de la innovación." }\end{array}$ & $\begin{array}{l}\text { González y } \\
\text { García }\end{array}$ & 2011 & 85 \\
\hline Innovación tradicional. & $\begin{array}{l}\text { "supone una perspectiva cerrada y } \\
\text { lineal de generación, desarrollo y } \\
\text { comercialización del conocimiento a } \\
\text { través de sus propias estructuras (...) } \\
\text { transferida de forma interna y se } \\
\text { previene su transferencia a los } \\
\text { competidores (...) el único } \\
\text { conocimiento estratégico es aquel } \\
\text { desarrollado a partir de fuentes } \\
\text { internas y, por el otro lado, que la } \\
\text { materialización de resultados } \\
\text { económicos se logra exclusivamente } \\
\text { a través de la incorporación de dicho } \\
\text { conocimiento en los productos de su } \\
\text { cartera." }\end{array}$ & $\begin{array}{l}\text { González } \\
\text { García }\end{array}$ & 2011 & 85 \\
\hline
\end{tabular}




\begin{tabular}{|c|c|c|c|c|}
\hline Nature of innovation. & $\begin{array}{l}\text { "refers to the form innovation as in } \\
\text { something new or improved." }\end{array}$ & Luoma et al. & 2012 & 11 \\
\hline $\begin{array}{l}\text { Organizational } \\
\text { innovativeness. }\end{array}$ & $\begin{array}{l}\text { "refer to the innovative abilities } \\
\text { needed whiting and organization } \\
\text { and among its employees. } \\
\text { Innovativeness is the overall internal } \\
\text { receptivity to new ideas and } \\
\text { innovation that is demonstrated } \\
\text { through individuals, teams and } \\
\text { management, and that enables the } \\
\text { formation and innovative culture." }\end{array}$ & Luoma et al. & 2012 & 10 \\
\hline $\begin{array}{l}\text { Organizational } \\
\text { performance. }\end{array}$ & $\begin{array}{l}\text { "is a measure of the efficiently and } \\
\text { effectively managers use available } \\
\text { resources to satisfy customers and } \\
\text { achieve organizational goals. } \\
\text { Organizational performance } \\
\text { increases in direct proportion to } \\
\text { increases in efficiency and } \\
\text { effectiveness". }\end{array}$ & $\begin{array}{l}\text { Abdiaziz y } \\
\text { Yassin }\end{array}$ & 2014 & 262 \\
\hline Organizational trust. & $\begin{array}{l}\text { "is a positive attitude held by one } \\
\text { organizational member toward } \\
\text { another the assumes that the order } \\
\text { party will act according to the rules of } \\
\text { fair play and with not take } \\
\text { advantage of one's vulnerability and } \\
\text { dependence in the risky situation." }\end{array}$ & Luoma et al. & 2012 & 13 \\
\hline psychological & $\begin{array}{l}\text { "Is a global construct that comprise } \\
\text { four different personal resources: } \\
\text { hope, resilience, optimism and } \\
\text { efficacy." }\end{array}$ & $\begin{array}{l}\text { Rodríguez et } \\
\text { al. }\end{array}$ & 2010 & 239 \\
\hline Positive Psychology. & $\begin{array}{l}\text { "The study an application of positively } \\
\text { oriented humans resource strengths } \\
\text { and psychological capacities that } \\
\text { can be measured, developed and } \\
\text { effectively managed for } \\
\text { performance improvement in today's } \\
\text { work place." }\end{array}$ & $\begin{array}{l}\text { Rodríguez et } \\
\text { al. }\end{array}$ & 2010 & 236 \\
\hline Psychological flexibility & $\begin{array}{l}\text { "individual is able to engaged to } \\
\text { present moment as a conscious } \\
\text { human being, and to act in accord } \\
\text { with his or her chosen values. This } \\
\text { results in being conscious of one's } \\
\text { own thoughts, and acting effectively } \\
\text { trough this consciousness. It enables } \\
\text { individuals to persist in or to the } \\
\text { change their actions according to } \\
\text { what they value as important, and } \\
\text { decreases rigid thinking and } \\
\text { behavior." }\end{array}$ & Luoma et al. & 2012 & 12 \\
\hline Psychological flexibility. & $\begin{array}{l}\text { "As being in contact with the preset } \\
\text { moment as a concious human being, } \\
\text { and, based on what that situation }\end{array}$ & $\begin{array}{l}\text { Rodríguez et } \\
\text { al. }\end{array}$ & 2010 & 240 \\
\hline
\end{tabular}




\begin{tabular}{|c|c|c|c|c|}
\hline & $\begin{array}{l}\text { affords, acting in accordance with } \\
\text { one chosen values. There are siz } \\
\text { processes involved: acceptance, } \\
\text { cognitive diffusion, being in the } \\
\text { present moment, self as context } \\
\text { values and commitment action" }\end{array}$ & & & \\
\hline Redefining/Restructuring. & $\begin{array}{l}\text { "stage the innovation is modified } \\
\text { and reinvented to suit the } \\
\text { organization". }\end{array}$ & Jalonen & 2012 & 4 \\
\hline Resources Theory. & $\begin{array}{l}\text { "we proposed that sustainable } \\
\text { competitive advantage result for } \\
\text { intangible assets because they } \\
\text { enable the accumulation of other } \\
\text { types of assets. Intangible assets are } \\
\text { inimitable, rare, and nontradable, } \\
\text { and include brand, knowledge, } \\
\text { flexible technology, personal skills, } \\
\text { contracts, and efficient procedures." }\end{array}$ & Luoma et al. & 2012 & 8 \\
\hline Retection. & $\begin{array}{l}\text { "Means the preservation or } \\
\text { duplication of selected ideas with the } \\
\text { result that they are repeated in the } \\
\text { future". }\end{array}$ & Jalonen & 2012 & 4 \\
\hline Routinizing. & $\begin{array}{l}\text { "the innovations becomes and } \\
\text { ongoing element in the organization's } \\
\text { every day life". }\end{array}$ & Jalonen & 2012 & 4 \\
\hline Selection. & $\begin{array}{l}\text { "Of new ideas is determined by the } \\
\text { interplay between organizational } \\
\text { competences and environmental } \\
\text { factors." }\end{array}$ & Jalonen & 2012 & 4 \\
\hline Social context & $\begin{array}{l}\text { "refers to any social entity, system or } \\
\text { group of people involved in the } \\
\text { innovation process or environmental } \\
\text { factor affecting." }\end{array}$ & Luoma et al. & 2012 & 11 \\
\hline $\begin{array}{l}\text { Sociopolitical } \\
\text { uncertainty }\end{array}$ & $\begin{array}{l}\text { "can result from a diversity of interest } \\
\text { among stakeholders and power } \\
\text { struggle between stakeholders". }\end{array}$ & Jalonen & 2012 & 27 \\
\hline Stages of innovation. & $\begin{array}{l}\text { "refers to all the steps taken during } \\
\text { and innovation process, which usually } \\
\text { star from idea generation and end } \\
\text { with commercialization." }\end{array}$ & Luoma et al. & 2012 & 11 \\
\hline Struggle. & $\begin{array}{l}\text { "Arises due to scarcity of resources } \\
\text { within organizations and between } \\
\text { them in a given environment." }\end{array}$ & Jalonen & 2012 & 4 \\
\hline Timing uncertainty. & $\begin{array}{l}\text { "in innovation results a lack of } \\
\text { information in the early phases of } \\
\text { innovation, from the ambiguity of } \\
\text { information in the late phases of } \\
\text { innovation or from temporal } \\
\text { complexity faced by innovators." }\end{array}$ & Jalonen & 2012 & 31 \\
\hline
\end{tabular}




\begin{tabular}{|l|l|l|l|l|}
\hline Tipe of innovation. & $\begin{array}{l}\text { "refers to the King of innovation, as in } \\
\text { the type of output of the result of } \\
\text { innovation." }\end{array}$ & 2012 & 11 \\
\hline Uncertainty technology. & $\begin{array}{l}\text { "arises due to lack of knowledge of } \\
\text { the details of new technology or due } \\
\text { to a lack of knowledge required to } \\
\text { use to technology." }\end{array}$ & Jalonen & 2012 & 24 \\
\hline Variations & $\begin{array}{l}\text { "refers to any intentional or or Jalonen } \\
\text { unintentional departure from routine. } \\
\text { Manifest themselves as new ideas, of } \\
\text { which some will be selected and } \\
\text { others eliminated". }\end{array}$ & 2012 & 4 \\
\hline
\end{tabular}

Tabla 2. Estado del conocimiento

\begin{tabular}{|c|c|c|c|c|}
\hline Año & Autor & Muestra & Instrumentos & Hallazgos \\
\hline $\begin{array}{l}201 \\
0\end{array}$ & $\begin{array}{l}\text { Rodríguez } \\
\text { et al. }\end{array}$ & $\begin{array}{l}65 \text { artículos de } 2005 \\
\text { a } 2010\end{array}$ & $\begin{array}{l}\text { Inventario de } \\
\text { documentaciónindexad } \\
\text { a }\end{array}$ & $\begin{array}{l}\text { La cultura } \\
\text { organización } \\
\text { está relacionada } \\
\text { diferencialmente } \\
\text { con la literatura } \\
\text { en torno a la } \\
\text { satisfacción en el } \\
\text { trabajo. Los } \\
\text { niveles de estrés, } \\
\text { y satisfacción no } \\
\text { determinan el } \\
\text { bienestar } \\
\text { laboral. } \\
\text { liderazgo } \\
\text { determina } \\
\text { satisfacción con } \\
\text { el empleo, ya } \\
\text { que una buena } \\
\text { relación } \\
\text { poder determina } \\
\text { un clima de } \\
\text { tareas y } \\
\text { relaciones con } \\
\text { los pares. } \\
\text { Transparencia y } \\
\text { optimismo fueron } \\
\text { los atributos } \\
\text { asignados a un } \\
\text { buen liderazgo. } \\
\text { La iniciativa y el } \\
\text { empoderamient } \\
\text { o son factores } \\
\text { del logro de } \\
\text { objetivos, el } \\
\text { crecimiento, la } \\
\text { auto-realización } \\
\text { y el bienestar. } \\
\text { Las atribuciones } \\
\text { ocupacionales }\end{array}$ \\
\hline
\end{tabular}









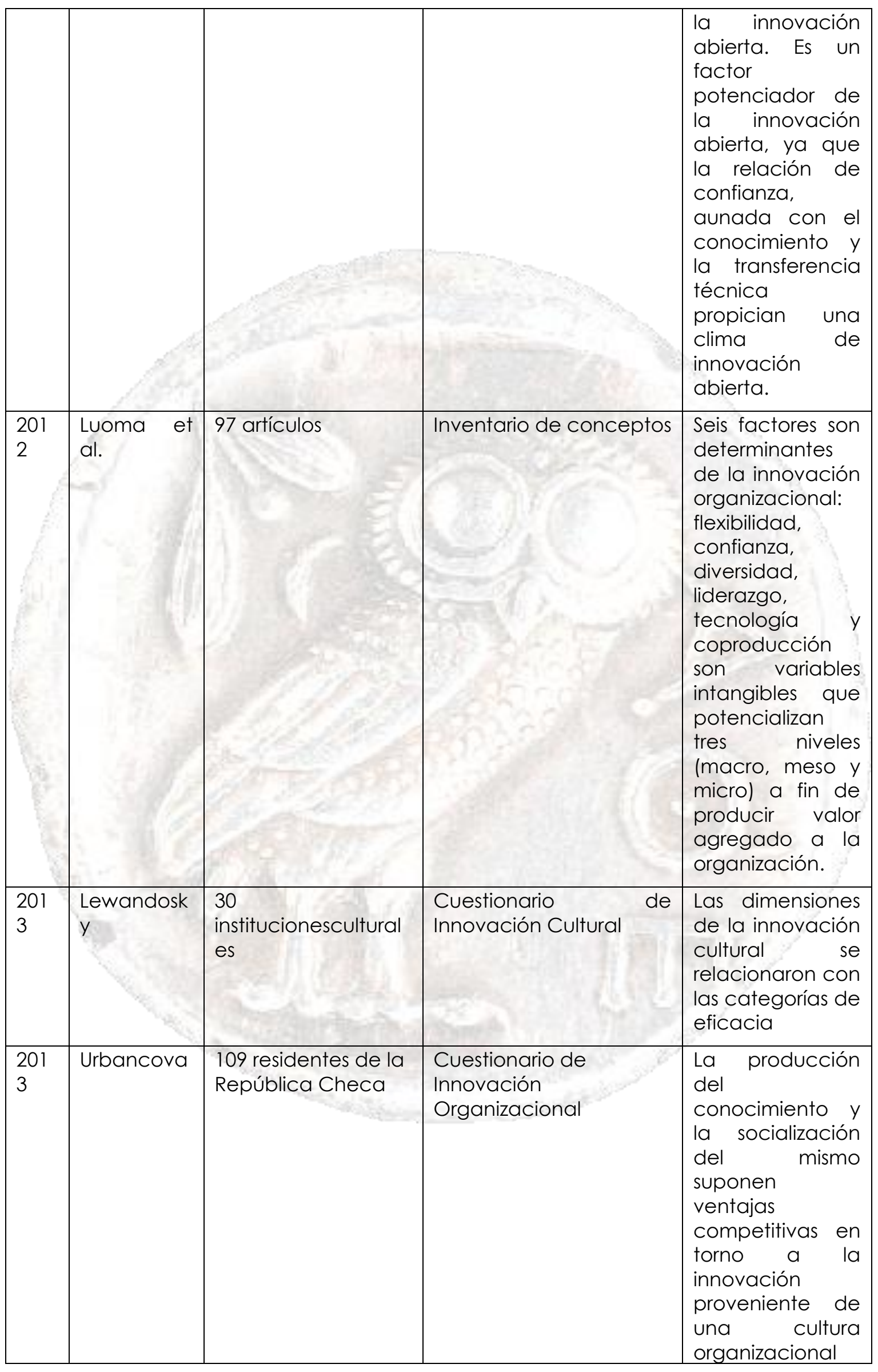




\begin{tabular}{|c|c|c|c|c|}
\hline & & & & flexible. \\
\hline $\begin{array}{l}201 \\
4\end{array}$ & $\begin{array}{l}\text { Abdiaziz y } \\
\text { Yassin }\end{array}$ & $\begin{array}{l}160 \text { empleados de } \\
\text { compañías de } \\
\text { telecomunicacione } \\
\mathrm{s}\end{array}$ & $\begin{array}{l}\text { Cuestionario de } \\
\text { Innovación del Entorno }\end{array}$ & $\begin{array}{l}\text { La } \\
\text { administración } \\
\text { innovativa } \\
\text { determinó el } \\
\text { desempeño } \\
\text { organizacional } \\
{[\beta=0,389 \quad(\dagger=} \\
4,252) p<0,001]\end{array}$ \\
\hline $\begin{array}{l}201 \\
4\end{array}$ & Marques & 40 artículos & $\begin{array}{l}\text { Inventario de } \\
\text { definiciones }\end{array}$ & $\begin{array}{l}\text { El concepto de } \\
\text { innovación } \\
\text { abierta al } \\
\text { plantear un ciclo } \\
\text { de aprendizaje } \\
\text { contraviene el } \\
\text { sistema de } \\
\text { mercado; la } \\
\text { apertura de las } \\
\text { innovaciones } \\
\text { supone la } \\
\text { intromisión de } \\
\text { agentes } \\
\text { desestabilizador } \\
\text { es u oportunistas } \\
\text { que deterioran } \\
\text { al sistema; } \\
\text { incompatibilidad } \\
\text { con la historia de } \\
\text { las } \\
\text { organizaciones y } \\
\text { la dinámica de } \\
\text { los mercados. }\end{array}$ \\
\hline $\begin{array}{l}201 \\
3\end{array}$ & $\begin{array}{l}\text { Rodríguez y } \\
\text { Hechanov } \\
\text { a }\end{array}$ & $\begin{array}{l}245 \text { empleados de } \\
\text { una compañía de } \\
\text { telecomunicacione } \\
\text { s }\end{array}$ & 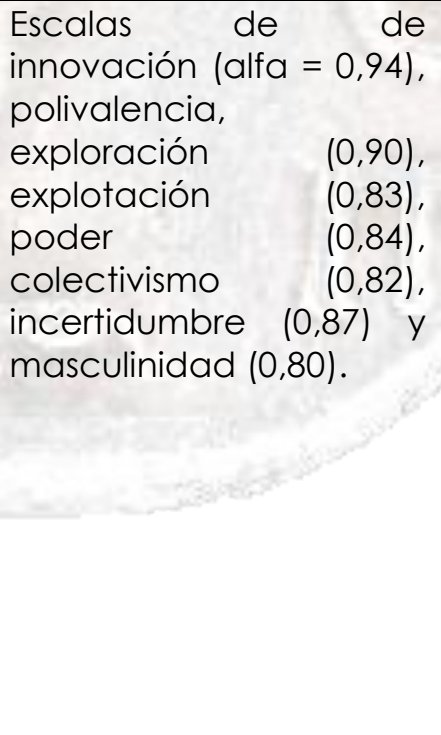 & $\begin{array}{l}\text { La polivalencia } \\
\text { se relacionó } \\
\text { positivamente } \\
\text { con la } \\
\text { exploración ( } r= \\
0,91 ; p<0,01) \text { y } \\
\text { ésta última con } \\
\text { lainnovación ( } r= \\
0,71 ; p<0,01) \text {, la } \\
\text { explotación se } \\
\text { vinculo con la } \\
\text { polivalencia ( } r= \\
0,85 ; p<0,01) \text {. La } \\
\text { innovación fue } \\
\text { determinada por } \\
\text { la polivalencia }(\beta \\
=0,61) \text {. }\end{array}$ \\
\hline
\end{tabular}

